\title{
Time-lapse travel time change of multiply scattered acoustic waves
}

\author{
Carlos Pacheco \\ Center for Wave Phenomena, Department of Geophysics, Colorado School of Mines, Golden, \\ Colorado 80401 \\ Roel Snieder \\ Center for Wave Phenomena, Department of Geophysics, Colorado School of Mines, Golden, \\ Colorado 80401
}

(Received 12 August 2004; revised 6 May 2005; accepted 27 June 2005)

\begin{abstract}
Existing techniques in correlation spectroscopy, such as coda wave interferometry and diffusing acoustic wave spectroscopy, determine the average motion of scatterers or change in the propagation velocity from the temporal change of multiply scattered sound. However, neither of them gives an indication of the spatial extent of the change in the medium. This study is an extension of the technique coda wave interferometry, where multiply scattered waves are used to determine the change in the wave field due to a localized perturbation in the propagation velocity. Here, the propagation of multiply scattered sound is described using the diffusion approximation, which allows the cross-correlation function of the unperturbed and perturbed wave fields to be related to the localized change in the propagation velocity. The technique is tested numerically for two-dimensional (2D) acoustic waves using synthetic seismograms calculated using finite-differences before and after a small perturbation in the propagation velocity has been introduced. Despite the relatively small size and magnitude of the change, multiple scattering greatly amplifies small perturbations, making changes in the phase or travel time of the wave field visible in the later-arriving waveforms. Potential applications of this technique include nondestructive evaluation of inhomogeneous materials and time-lapse monitoring of volcanoes and highly heterogeneous reservoirs. (C) 2005 Acoustical Society of America. [DOI: 10.1121/1.2000827]
\end{abstract}

PACS number(s): 43.20.Fn, 43.28.Lv, 43.40.Ph [LLT]

Pages: $1300-1310$

\section{INTRODUCTION}

Most imaging techniques using scattered waves rely on the single scattering approximation. Seismic imaging ${ }^{1}$ mostly uses primary reflected waves to obtain an image of the subsurface. In many physical problems, however, waves are strongly scattered and the single scattering approximation is not a valid model for the propagation of waves through the medium. In such cases we have to use a model that accounts for the multiple scattering of waves and the associated attenuation. The diffusion model has been used with success to characterize a wide range of wave phenomena in strongly scattering media. ${ }^{2-6}$ In this model, wave energy transport acquires a diffusive character, e.g., wave energy is transported in a process similar to heat diffusion. In medical imaging, for example, diffusing near-infrared light has been used to image localized heterogeneities of tissue. ${ }^{7}$

In many practical applications the medium changes over time, and therefore the image of the medium changes. We then would like to obtain time-lapse measurements in order to monitor temporal changes in the medium. Examples of applications where detecting temporal changes may be useful include the monitoring of volcanoes, oil reservoirs, radioactive waste disposal sites, and fluidized suspensions. In reflection seismology, dynamic reservoir characterization provides optimal management of a reservoir, which leads to increased production. Time-lapse (4D) reflection seismic aims at inferring changes from the medium from changes in the seismic amplitudes and/or travel times from seismic reflection data that have been acquired at two different times. As an example, a 4D dataset recorded at Weyburn Field, Canada, has been used to infer time-lapse changes in the oil reservoir caused by a massive miscible $\mathrm{CO}_{2}$ flood to enhance oil recovery. ${ }^{8,9}$ The main goal of these $4 \mathrm{D}$ studies is to extract information about local changes in the reservoir using mainly the amplitude information.

The fine structure of strongly scattered waves can provide a wealth of new information in seismology, ultrasonics, acoustics, and other fields that study wave propagation in heterogeneous media. ${ }^{10}$ Multiple scattering theory has been used to determine the number and the scattering strength of scatterers in motion inside a highly reflecting cavity, and more specifically for fish counting in a tank. ${ }^{11,12}$ In biophysics and medical imaging, diffusing photons are now used to view body function and structure after it was found that photon transport within tissues is dominated by scattering rather than absorption. ${ }^{7}$ Diffuse transmission spectroscopy ${ }^{13}$ has been used to probe the structure of opaque materials such as colloids, foams, and sand, using multiply scattered photons.

Multiply scattered waves have also been used to study the dynamics of complex media and turbulent fluids. Diffuse light spectroscopy ${ }^{7}$ has been used to measure the spatial variations in the absorption and scattering of large tissue volumes. Diffusing wave spectroscopy (DWS) is a technique in which multiply scattered light is used to study the dynamics 
of colloidal suspensions; ${ }^{14}$ this technique was adapted later to acoustic waves. ${ }^{15}$ This technique estimates the average motion of the scatterers from the temporal fluctuations of multiply scattered sound. In this technique, the propagation of multiply scattered sound is described using the diffusion equation, which makes it possible to relate the temporal field fluctuations with the dynamics of the multiple scattering medium. Recently, coda waves have been used to study the temperature dependence of the seismic velocity in granite ${ }^{16}$ and in a reverberant three-dimensional cavity ${ }^{17}$ using a technique called coda wave interferometry. In this technique, multiply scattered waves are used to detect temporal changes in a medium by using the scattering medium as an interferometer. For small changes in the medium, estimates of this perturbation can be obtained from multiply scattered waves by a cross correlation in the time domain. ${ }^{16}$ For larger changes, the decay of the correlation of the diffuse field is related to distortion and is an interesting system-dependent quantity. ${ }^{17}$ Coda wave interferometry has also been used to determine the relative location of earthquakes for scattered waves. $^{18}$

In none of those approaches has an attempt been made to determine the spatial extent of the change or local perturbation in the medium. When the random multiple scattering medium changes over time, changes in the diffuse field are related to changes in the medium. The sensitivity to perturbations in the medium enhances as the scattering order increases and thus becomes an interesting tool to detect the appearance of a very small defect in a multiple scattering sample. ${ }^{19}$ In this study we develop a technique that relates the localized changes in the propagation velocity of the medium to the travel time change of the multiply scattered waves. As in diffuse wave spectroscopy, this technique relies on the diffusion approximation of the intensity in strongly scattering media. Therefore, we can model the mean travel time change of waves with propagation time $t$ before and after a small and localized change in the propagation velocity has been introduced in the medium. This work is an extension of coda wave interferometry in the sense that it accounts for localized changes in the propagation velocity of the scattering medium. Thus, we are able to model the fluctuation in the phase of the multiply scattered wave field for a given localized time-lapse velocity perturbation of the multiple scattering medium. We assess the validity of our theory using finite-difference simulations of multiple scattering of acoustic waves in $2 \mathrm{D}$ media.

The paper is divided into five parts. Section II explains how the diffusion approximation is used to describe energy transport in strongly scattering media and its relation with a random walk process. Section III introduces coda wave interferometry and how it can be used to obtain an estimator for the mean travel time change of scattered waves. In Sec. IV we derive theoretically an expression for the mean travel time change of multiply scattered waves by using the analogy between a diffusion process and random walks. The main result of this work is the expression that relates the mean travel time change of multiply scattered waves with the localized perturbation in the propagation velocity of the medium. In Sec. V we validate the theory with numerical ex-
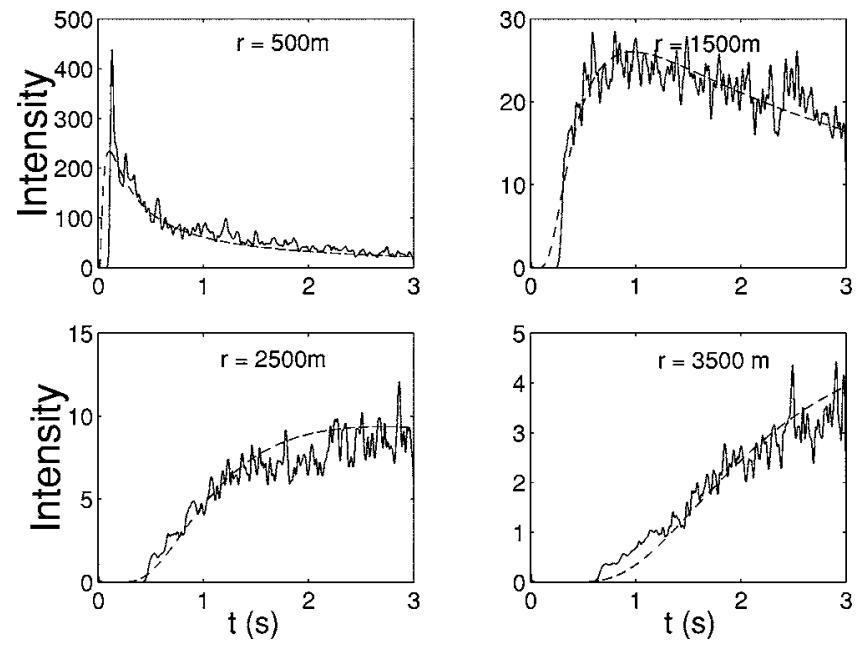

FIG. 1. Actual averaged intensities (solid line) versus best diffusion fit (dashed line) for receivers at 500, 1500, 2500, and $3500 \mathrm{~m}$. The estimated value of the diffusion constant is $5.78 \times 10^{5} \mathrm{~m}^{2} / \mathrm{s}$.

amples using finite-difference synthetic seismograms. We found good agreement between our theory and results from the finite-difference seismograms, despite some fluctuations that are analyzed in Sec. VI. Finally, we discuss applications and limitations of the technique in Sec. VII.

\section{DIFFUSION ENERGY TRANSPORT AND RANDOM WALKS}

The transport of energy through a strongly scattering medium has attracted considerable attention in numerous fields of physics, such as astrophysics, optics, acoustics, solid-state physics, and heat conduction. In any of these fields, one studies a pulse of energy that propagates through the medium with an intensity $P(r, t)$. The diffusion equation describes the propagation of the average intensity in a multiple scattering medium. ${ }^{20}$ In a two-dimensional medium of infinite extent, constant scattering properties, without intrinsic attenuation, and in the long-time limit, ${ }^{21}$ the average intensity at $\mathbf{r}$ can be approximated by the solution of the diffusion equation

$$
P(\mathbf{r}, t)=\frac{1}{4 \pi D t} \exp \left[\frac{-r^{2}}{4 D t}\right],
$$

where $r$ is the distance to the source and $D$ is the diffusion constant. Equation (1) describes the temporal evolution of the average intensity after the waves have scattered multiple times from small-scale heterogeneities. Figure 1 shows the average intensity for receivers located 500, 1500, 2500, and $3500 \mathrm{~m}$ away from the source. The actual mean intensities were calculated after averaging over 100 different receiver locations after propagating a seismic wave field through a medium with random velocity fluctuations using finite differences as described in Sec. V. Figure 2 shows one of the seismograms calculated for a source-receiver distance of $2500 \mathrm{~m}$. Despite the random appearance of the individual seismogram, an ensemble of such seismograms yields an average intensity that obeys the diffusion equation. The mean intensities from the finite-difference simulations are shown as solid curves, whereas the best diffu- 


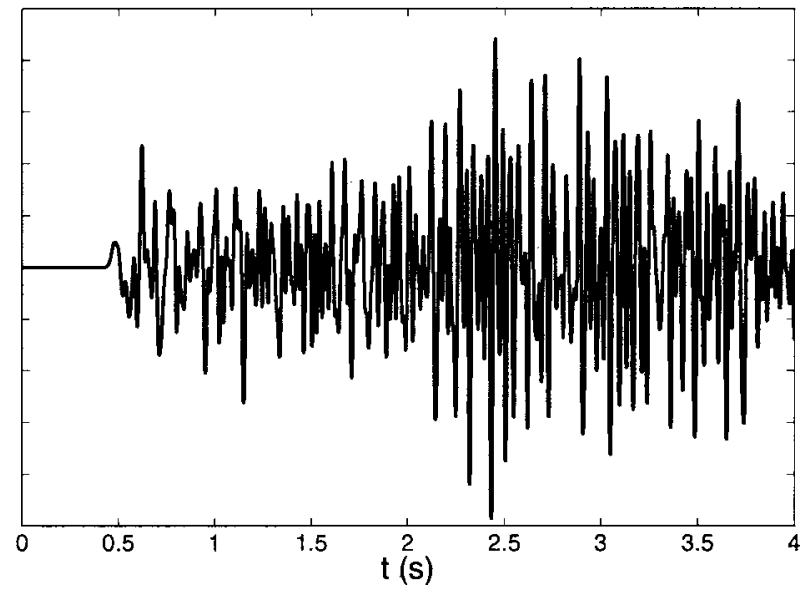

FIG. 2. Example of a finite-difference seismogram for a source-receiver distance of $2500 \mathrm{~m}$ after propagating a finite-bandwidth pulse through a medium with random velocity fluctuations.

sion fits [Eq. (1)] are shown as dashed lines. Notice the good agreement between the diffusion curves and the average intensities.

Given a seismogram such as the one shown in Fig. 2, it is extremely difficult to determine if a specific trajectory gives rise to an energy impulse at a specific time $t$. The problem is simplified if, instead of considering one specific trajectory, we consider the average wave field obtained after summing the contributions of all possible trajectories arriving at the receiver at time $t$. When scattering is strong and waves follow infinitely many trajectories, wave propagation can be considered as a random walk process. This simplifies the problem because a random walk process can on average be described with the solution of the diffusion equation. This provides us with the advantage of a simple physical picture where the diffusively scattered wave field is represented as the sum of partial waves traveling along various diffuse paths. ${ }^{22}$

The multiply scattered waves travel through the medium in a random walk process that is characterized in $n$-D (where $n$ is the number of dimensions) by the transport mean free path $l^{*}$, the energy velocity $v_{e}$, and the diffusion coefficient $D=v_{e} l^{*} / n{ }^{15}$ Figure 3 shows three realizations of random

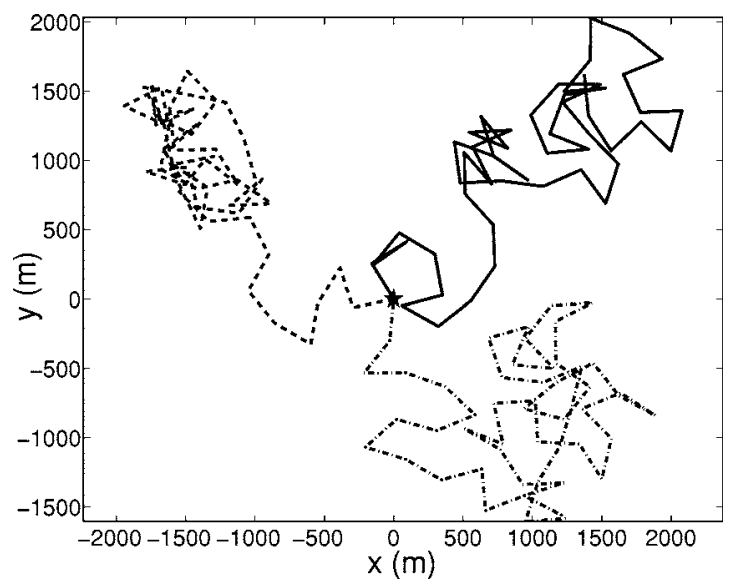

FIG. 3. Three realizations of the random walk characterized by $D=3.45$ $\times 10^{5} \mathrm{~m}^{2} / \mathrm{s}$ and $v_{e}=5300 \mathrm{~m} / \mathrm{s}$.

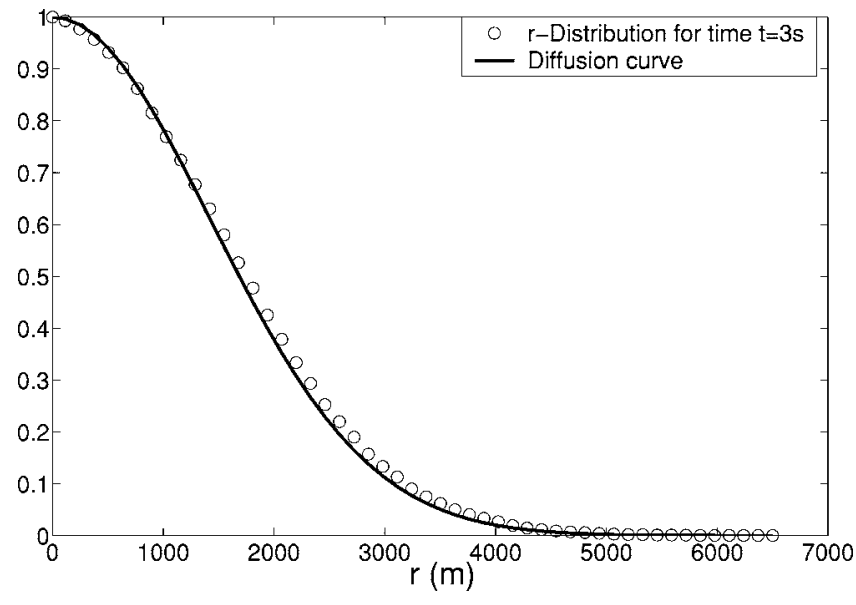

FIG. 4. Observed distribution of distances for the 5000 realizations of random walks (open circles) versus the diffusion curve (solid line) for $t=3 \mathrm{~s}$.

walk in 2D calculated numerically using $D=3.45$ $\times 10^{5} \mathrm{~m}^{2} / \mathrm{s}$ and $v_{e}=5300 \mathrm{~m} / \mathrm{s}$. The random walks were calculated until a maximum elapsed time $t=3 \mathrm{~s}$. If we calculate many realizations, the distribution of distances $r$ traveled from the origin at a given time $t$ is given by Eq. (1). This is shown in Fig. 4, were we show the distribution of distances traveled for the random walk particles for 5000 different realizations of random walks starting from the origin with total elapsed time $t=3 \mathrm{~s}$. In Sec. IV we use the random walk approximation to multiple scattering to derive an expression for the mean travel time of waves in a strongly scattering medium.

\section{CODA WAVE INTERFEROMETRY}

When a strongly scattering medium changes, the speckle pattern of multiply scattered waves changes, which reflects the changes that occur in the interference of waves traveling with different scattering paths through the sample. Multiply scattered waves are useful in such situations, because they are increasingly sensitive with time to the perturbations in scatterer locations or perturbations in the propagation velocity of the medium. This increased sensitivity is due to the fact that waves bounce more often among scatterers as time increases, and as a result, small changes in the medium are amplified through multiple scattering.

For a small perturbation in the propagation velocity, an estimate of this perturbation can be obtained by a timewindowed cross correlation of the unperturbed and perturbed scattered waves. ${ }^{23}$ The unperturbed wave field $u_{\text {unp }}(t)$ can be written as a summation of waves over all possible trajectories $T^{24}$

$$
u_{\text {unp }}(t)=\sum_{T} A_{T}(t),
$$

where a trajectory $T$ is defined by the sequence of scatterers that a particular multiple scattering wave encounters, and $A_{T}(t)$ is the corresponding waveform. For diffusive wave propagation the trajectories $T$ can be thought of as a collection of random walks.

C. Pacheco and R. Snieder: Monitoring with multiply scattered waves 
When we introduce a small perturbation of the propagation velocity, the dominant effect on the multiple scattering waveform is a travel time perturbation $\tau_{T}$ of the wave that travels along the trajectory $T$

$$
u_{\mathrm{per}}(t)=\sum_{T} A_{T}\left(t-\tau_{T}\right) .
$$

We can characterize the change in the wave field using the time-windowed cross-correlation function ${ }^{23}$

$$
C_{\mathrm{up}}^{\left(t, t_{w}\right)}\left(t_{s}\right)=\int_{t-t_{w}}^{t+t_{w}} u_{\mathrm{unp}}\left(t^{\prime}\right) u_{\mathrm{per}}\left(t^{\prime}+t_{s}\right) d t^{\prime},
$$

where $t$ denotes the center of a time window of length $2 t_{w}$, and $t_{s}$ is the lag time for the correlation. When Eqs. (2) and (3) are inserted into Eq. (4), double sums $\Sigma_{T T^{\prime}}$ appear. We assume that in these double sums, the cross terms related to different trajectories $\left(T \neq T^{\prime}\right)$ are incoherent and average out to zero when the mean of the source signal vanishes. The contribution of the cross terms is estimated in Ref. 25. A dimensionless measure of the change of the wave field is given by the time-windowed correlation coefficient, which is given by

$$
R^{\left(t, t_{w}\right)}\left(t_{s}\right)=\frac{\int_{t-t_{w}}^{t+t_{w}} u_{\mathrm{unp}}\left(t^{\prime}\right) u_{\mathrm{per}}\left(t^{\prime}+t_{s}\right) d t^{\prime}}{\left(\int_{t-t_{w}}^{t+t_{w}} u_{\mathrm{unp}}^{2}\left(t^{\prime}\right) d t^{\prime} \int_{t-t_{w}}^{t+t_{w}} u_{\mathrm{per}}^{2}\left(t^{\prime}\right) d t^{\prime}\right)^{1 / 2}} .
$$

For time shifts $t_{s}$ much smaller than the dominant period of the wave, a second-order Taylor expansion of the $A_{T}\left(t^{\prime}\right.$ $\left.+t_{s}-\tau_{T}\right)$ in $\tau_{T}$ gives $^{23}$

$$
R^{\left(t, t_{w}\right)}\left(t_{s}\right)=1-\frac{1}{2} \bar{\omega}^{2}\left\langle\left(\tau-t_{s}\right)^{2}\right\rangle_{\left(t, t_{w}\right)},
$$

where $\bar{\omega}$ is the dominant frequency of the wave. In this expression $\langle\ldots\rangle_{\left(t, t_{w}\right)}$ denotes the average over all waves that arrive in the time window $\left(t-t_{w}, t+t_{w}\right)$ with a weight factor that is equal to the intensity of the waves. ${ }^{23}$ Thus, averages are taken with a weight factor that is given by the intensity of each wave. This means that in this work, the average travel time change $\langle\tau\rangle_{\left(t, t_{w}\right)}$ is given by an average of the travel time change of individual waves with different trajectories $T$ arriving on the time window $\left(t-t_{w}, t+t_{w}\right)$, i.e.,

$$
\langle\tau\rangle_{\left(t, t_{w}\right)}=\frac{\sum_{T} w_{T}\left(t, t_{w}\right) \tau_{T}\left(t, t_{w}\right)}{\sum_{T} w_{T}\left(t, t_{w}\right)},
$$

where the weighting factor $w_{T}\left(t, t_{w}\right)$ is given by the intensity for the diffuse waves arriving on the time window $\left(t-t_{w}, t\right.$ $\left.+t_{w}\right)$.

\section{MEAN TRAVEL TIME CHANGE IN THE DIFFUSION REGIME}

\section{A. Random walk probabilities}

Each random walk from the source to the receiver has an associated probability which depends on the diffusion of the intensities in the strongly scattering medium. Recalling Eq. (7), and considering waves arriving on the time window ( $t$

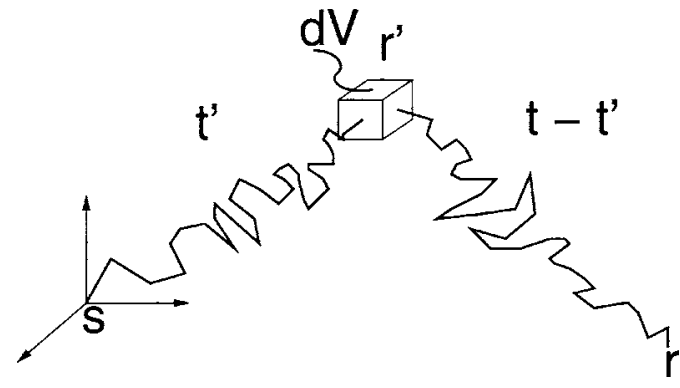

FIG. 5. A random walk particle going from source $\mathbf{s}$ at the origin to volume element $d V$ at $\mathbf{r}^{\prime}$ on time $t^{\prime}$, and then to receiver at $\mathbf{r}$ on time $t-t^{\prime}$.

$\left.-t_{w}, t+t_{w}\right)$, the average or mean travel time change $\langle\tau\rangle$ is a weighted average of the travel time changes of the individual trajectories $T$, i.e.,

$$
\langle\tau\rangle=\frac{\sum_{T} I_{T} \tau_{T}}{\sum_{T} I_{T}},
$$

where $I_{T}$ is the intensity or probability associated with the trajectory $T$, which can be calculated using the solution to the diffusion equation. In the following, we show how to derive an expression for this intensity for a given source and receiver location.

Let us assume that the seismic energy transport can be described as a diffusion process. Thus, the space and time evolution of the diffusive intensity in the medium due to an intensity impulse at the origin at time $t=0$ is given by $P(\mathbf{r}, t)$, as defined in Eq. (1). In multiple scattering of waves, this diffusive energy corresponds to the ensemble averaged or mean intensity $\langle I\rangle$.

We can interpret $P(\mathbf{r}, t)$ in a different way, regarding diffusion as a random walk process. In random walk theory, the product $P \times d V$ represents the probability of a particle on a random walk of visiting a volume element $d V$ at location $\mathbf{r}$ at a given time $t .^{26}$ If, at time $t=0$, a normalized intensity impulse is generated at the source, the total energy within some region $V$ at some later time is given by the integral

$$
W(V, t)=\int_{V} P(r, t) d V(r) .
$$

Integration over all space gives the total energy of the system, which by the normalization is $W(t)=1$. The quantity $W(V, t)$ is equal to the probability of a particle on a random walk of visiting the volume region $V$ at a time $t$.

We now consider the probability that a random walk particle leaves a source at $\mathbf{s}$ at time $t=0$, visits a volume element $d V$ at $\mathbf{r}^{\prime}$ at time $t^{\prime}$, and arrives at $\mathbf{r}$ at time $t$ as depicted in Fig. 5. Assuming that the two paths, from source to the volume element, and from the volume element to the receiver, are independent, this probability is equal to the product of two probabilities: the probability of the particle of going from $\mathbf{s}$ to $\mathbf{r}^{\prime}$ in a time $t^{\prime}$, and the probability of going from $\mathbf{r}^{\prime}$ to $\mathbf{r}$ in a time $t-t^{\prime}$, i.e., 


$$
P\left(\mathbf{r}^{\prime}, \mathbf{r}, \mathbf{s}, t^{\prime}, t\right)=P\left(\mathbf{r}^{\prime}, \mathbf{s}, t^{\prime}\right) P\left(\mathbf{r}, \mathbf{r}^{\prime}, t-t^{\prime}\right),
$$

where $P\left(\mathbf{r}^{\prime}, \mathbf{r}, \mathbf{s}, t^{\prime}, t\right)$ stands for the probability of all the trajectories visiting the volume element at location $\mathbf{r}^{\prime}$ at time $t^{\prime}$ given that the walk started at the source location $\mathbf{s}$ and ended at the receiver location $\mathbf{r}$ at time $t$.

The probability of a particle to travel from the source to the receiver is given by the solution to the diffusion equation for homogeneous medium given in Eq. (1). This solution also gives the time-dependent intensity $P(\mathbf{r}, \mathbf{s}, t)$ at the receiver location due to a unit intensity impulse at the source. This intensity is obtained by summing the contributions to the intensity of all waves traveling with all possible diffuse trajectories going from the source to the receiver, i.e., integrating Eq. (10) over all possible volume elements $d V\left(\mathbf{r}^{\prime}\right)$

$$
\begin{aligned}
P(\mathbf{r}, \mathbf{s}, t) & =\int_{V} P\left(\mathbf{r}^{\prime}, \mathbf{r}, \mathbf{s}, t^{\prime}, t\right) d V\left(\mathbf{r}^{\prime}\right) \\
& =\int_{V} P\left(\mathbf{r}, \mathbf{r}^{\prime}, t-t^{\prime}\right) P\left(\mathbf{r}^{\prime}, \mathbf{s}, t^{\prime}\right) d V\left(\mathbf{r}^{\prime}\right) .
\end{aligned}
$$

The integrand $P\left(\mathbf{r}^{\prime}, \mathbf{r}, \mathbf{s}, t^{\prime}, t\right)$ contains the contributions to the intensity of all paths which are initiated at the source location $\mathbf{s}$, visit the location $\mathbf{r}^{\prime}$, and end at the receiver located at $\mathbf{r}$. By integrating over all the volume where scattering occurs, we are summing the contributions over all possible trajectories from the source to the receiver. Note that in Eq. (11) no integration over $t^{\prime}$ is performed. Equation (11) is a restatement of the Chapman-Kolmogorov equation ${ }^{26}$

$$
\int_{V} P\left(\mathbf{r}, \mathbf{r}^{\prime}, t\right) P\left(\mathbf{r}^{\prime}, \mathbf{s}, t^{\prime}\right) d V\left(\mathbf{r}^{\prime}\right)=P\left(\mathbf{r}, \mathbf{s}, t+t^{\prime}\right),
$$

which states that a random walk process starting at $t=0$ at location $\mathbf{s}$ reaches $\mathbf{r}$ at $t$ via one of the possible values $\mathbf{r}^{\prime}$ at a intermediate time $t^{\prime}$

The quantity $P\left(\mathbf{r}^{\prime}, \mathbf{s}, t^{\prime}\right)$ in Eq. (11) is the diffuse intensity at a time $t^{\prime}$ at $\mathbf{r}^{\prime}$ due to an impulse source at $\mathbf{s}$ activated at time $t=0$, and $P\left(\mathbf{r}, \mathbf{r}^{\prime}, t-t^{\prime}\right)$ is the intensity at $\mathbf{r}$ at time $t$ due to an impulse source at $\mathbf{r}^{\prime}$ on a time $t-t^{\prime}$. Equation (11) holds for all times $0<t^{\prime}<t^{26}$ If we integrate both sides of Eq. (11) over time $t^{\prime}$ over the interval $0<t^{\prime}<t$, we obtain

$$
t P(\mathbf{r}, \mathbf{s}, t)=\int_{V} \int_{0}^{t} P\left(\mathbf{r}, \mathbf{r}^{\prime}, t-t^{\prime}\right) P\left(\mathbf{r}^{\prime}, \mathbf{s}, t^{\prime}\right) d t^{\prime} d V\left(\mathbf{r}^{\prime}\right),
$$

where we can identify the time integral $\int_{0}^{t} P\left(\mathbf{r}, \mathbf{r}^{\prime}, t\right.$ $\left.-t^{\prime}\right) P\left(\mathbf{r}^{\prime}, \mathbf{s}, t^{\prime}\right) d t^{\prime}$ as the time convolution of the two intensities: one at location $\mathbf{r}^{\prime}$ due to an impulse intensity at $\mathbf{s}$ and the other at location $\mathbf{r}$ due to an impulse intensity at $\mathbf{r}^{\prime}$. If we divide both sides of Eq. (13) by $P(\mathbf{r}, \mathbf{s}, t)$, we arrive at the following integral representation for the travel time of the diffuse wave field:

$$
t=\frac{1}{P(\mathbf{r}, \mathbf{s}, t)} \int_{V} \int_{0}^{t} P\left(\mathbf{r}, \mathbf{r}^{\prime}, t-t^{\prime}\right) P\left(\mathbf{r}^{\prime}, \mathbf{s}, t^{\prime}\right) d t^{\prime} d V\left(\mathbf{r}^{\prime}\right) .
$$

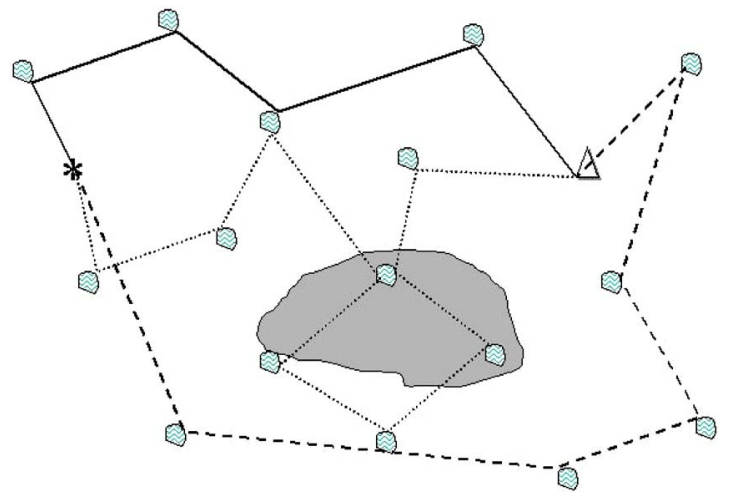

FIG. 6. (Color online) Sensitivity kernel $K$ in 2D for source (asterisk) and receiver (triangle) separated $3000 \mathrm{~m}$ and $t=2 \mathrm{~s}$. The diffusion constant is $5.8 \times 10^{5} \mathrm{~m}^{2} / \mathrm{s}$

We have obtained in Eq. (14) a representation for the time $t$ at $\mathbf{r}$ in terms of the diffuse intensity due to an impulse source at $\mathbf{s}$. The time $t$ corresponds to the time of propagation of the diffuse intensity. If we define the kernel $K\left(\mathbf{r}^{\prime}, \mathbf{r}, \mathbf{s}, t\right)$ as

$$
K\left(\mathbf{r}^{\prime}, \mathbf{r}, \mathbf{s}, t\right)=\frac{1}{P(\mathbf{r}, \mathbf{s}, t)} \int_{0}^{t} P\left(\mathbf{r}, \mathbf{r}^{\prime}, t-t^{\prime}\right) P\left(\mathbf{r}^{\prime}, \mathbf{s}, t^{\prime}\right) d t^{\prime},
$$

we can express the travel time $t$ as the following volume integral:

$$
t=\int_{V} K\left(\mathbf{r}^{\prime}, \mathbf{r}, \mathbf{s}, t\right) d V\left(\mathbf{r}^{\prime}\right),
$$

where $K\left(\mathbf{r}^{\prime}, \mathbf{r}, \mathbf{s}, t\right)$ represents the time of flight distribution of multiply scattered waves started at the source at location $\mathbf{s}$, visiting location $\mathbf{r}^{\prime}$ and detected on a receiver at location $\mathbf{r}$. Figure 6 shows a plot of the sensitivity kernel calculated for a source and receiver separated $3000 \mathrm{~m}, t=1 \mathrm{~s}$, and $D=5.8$ $\times 10^{5} \mathrm{~m}^{2} / \mathrm{s}$. Note that the kernel vanishes outside the area of an ellipse with foci at the source and receiver location.

\section{B. Integral representation for the mean travel time change of the diffuse wave field}

When the scatterers in a multiple scattering material move, or when the propagation velocity of the medium changes, the diffuse wave field changes. In diffusing acoustic wave spectroscopy, ${ }^{15}$ the fluctuations of the multiply scattered wave field are measured and analyzed to provide a sensitive technique for probing the dynamics of the scatterers. Here, we use a similar approach, considering the phase changes in the diffuse wave field which arise due to a spatially localized change in the propagation velocity.

We perturb the slowness in a finite region of the medium as shown in Fig. 7. We work under the assumption that the perturbation is weak so that the scattering coefficient does not change, and the waveform for each scattering path stays approximately the same. Also, the scattering paths remain unchanged so that the only difference between the unperturbed and the perturbed field is a small travel time perturbation, $\tau_{T}$. If the mean slowness of the medium is denoted by 


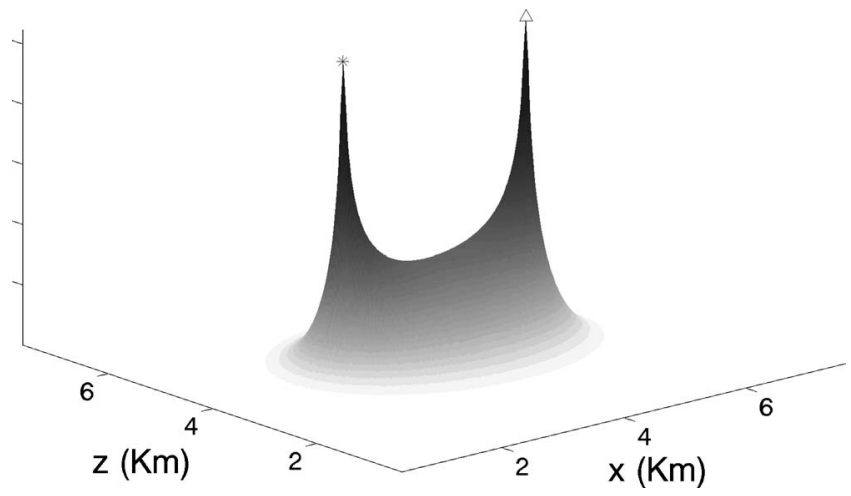

FIG. 7. Multiple scattering paths and a localized perturbation. After the perturbation the paths remain the same. The source location is represented by an asterisk and the receiver by a triangle.

$s$, we calculate the mean length $\langle L(t)\rangle$ of the multiple scattering paths at time $t$ by dividing Eq. (16) by $s$

$$
\langle L(t)\rangle=\int_{V} \frac{1}{S} K\left(\mathbf{r}^{\prime}, t\right) d V\left(\mathbf{r}^{\prime}\right),
$$

where we have placed $s$ inside the integral since it is assumed to be independent of location. For notation simplicity we have removed the explicit dependence of $K$ on the source and receiver location. A perturbation in the slowness gives rise to a perturbation in the travel time or phase of the wave field, i.e.,

$$
t+\delta_{t}=\int_{V} K\left(\mathbf{r}^{\prime}, t\right) \frac{\left(s+\delta_{s}\right)}{s}\left(\mathbf{r}^{\prime}\right) d V\left(\mathbf{r}^{\prime}\right),
$$

where $\delta_{s}$ is the perturbation in the background slowness. Since in the integral in Eq. (18) we are averaging the contribution to the travel time perturbation of all volume elements, $\delta_{t}$ becomes the average or mean travel time change that we define as $\langle\tau(t)\rangle$. We have assumed that the perturbation in the slowness is small $(\delta s / s \ll 1)$ so that the travel time perturbation depends only linearly on the slowness perturbation. The average or mean travel time change for the multiple scattering paths with path length $L$ is then

$$
\langle\tau(t)\rangle=\int_{V} K\left(\mathbf{r}^{\prime}, t\right) \frac{\delta s}{s}\left(\mathbf{r}^{\prime}\right) d V\left(\mathbf{r}^{\prime}\right),
$$

where $\langle\tau(t)\rangle$ is the mean travel time change of the multiply scattering waves with travel time $t$ and path length $\langle L(t)\rangle$ due to the relative slowness perturbation $\delta s / s$. Note that this average is weighted by the intensity, since the integration kernel $K\left(\mathbf{r}^{\prime}, t\right)$ represents the intensity of diffuse trajectories going through location $\mathbf{r}^{\prime}$ with total travel time $t$, as needed in the averaging in Eq. (8).

To calculate the mean travel time change for a particular source and receiver configuration, we need to integrate the kernel $K$ weighted by the slowness perturbation $\delta s / s$ over the volume where scattering takes place. The kernel $K$ is given by the time convolution in Eq. (15). For a source and receiver at different locations, this time convolution does in general not have an analytical solution so it must be evaluated numerically. For the special case of coincident source and receiver, an analytical solution for this convolution can be obtained. In the Appendix we calculate the kernel $K$ for coincident source and receiver for two- and threedimensional media. In 3D, the kernel $K$ is given by

$$
K_{3 D}(\mathbf{r}, t)=\frac{1}{2 \pi D r} \exp \left[\frac{-r^{2}}{D t}\right] .
$$

From Eq. (20) we see that the main contributions to the travel time change come from paths located close to the coincident source and receiver location. Also, we can see that for a fixed distance $r$ the integration kernel $K$ increases with time $t$. In multiple scattering, the effective distance traveled by diffuse waves is proportional to the square root of distance $(r \sim \sqrt{4 D t})$. For smaller value of the diffusion coefficient, the stronger the scattering is and the smaller the effective distance traveled by diffuse waves (wave paths become more localized around the source and receiver location). Thus, with increased time, diffuse waves sample the same region multiple times and consequently the travel time change increases with time.

For the special case of 2D wave propagation the integration kernel $K$ is for coincident source and receiver (see the Appendix for derivation)

$$
K_{2 D}(\mathbf{r}, t)=\frac{1}{2 \pi D} \exp \left[\frac{-r^{2}}{2 D t}\right] K_{0}\left[\frac{r^{2}}{2 D t}\right],
$$

where $K_{0}$ is the modified Bessel function of the second kind. If we insert Eq. (21) into Eq. (19) and integrate over area instead of volume, we obtain

$$
\langle\tau(t)\rangle=\frac{1}{2 \pi D} \int_{A} \exp \left[\frac{-r^{2}}{2 D t}\right] K_{0}\left[\frac{r^{2}}{2 D t}\right] \frac{\delta s}{s}(\mathbf{r}) d A(\mathbf{r}),
$$

where $r$ is the distance from the slowness perturbation $\delta s / s(\mathbf{r})$ element to the coincident source and receiver location. In Eq. (22), we have obtained an explicit expression relating the mean travel time change $\langle\tau\rangle$ of the diffuse waves to the localized slowness perturbation $[\delta s / s(\mathbf{r})]$ in a multiple scattering medium for coincident source and receiver. In general, for a given perturbation in slowness, we can predict the mean travel time change $\langle\tau\rangle$ for any source and receiver configuration by numerically calculating the convolution in Eq. (19).

\section{TRAVEL TIME CHANGE FROM FINITE-DIFFERENCE SYNTHETICS}

To generate synthetic seismograms for our study of multiple scattering, we use a fourth-order 2D acoustic finitedifference code that propagates a finite-duration pulse through a specified velocity field. Following Ref. 27, we model the 2D velocity field as a constant-background model with added random velocity fluctuations that constitute the scatterers (see Fig. 8). The total velocity field can be decomposed as

$$
v(\mathbf{r})=v_{0}+v_{r}(\mathbf{r}),
$$

where $v_{0}$ is the background velocity and $v_{r}$ are the random velocity fluctuations. The velocity fluctuations are characterized by a Gaussian autocorrelation function with correlation 


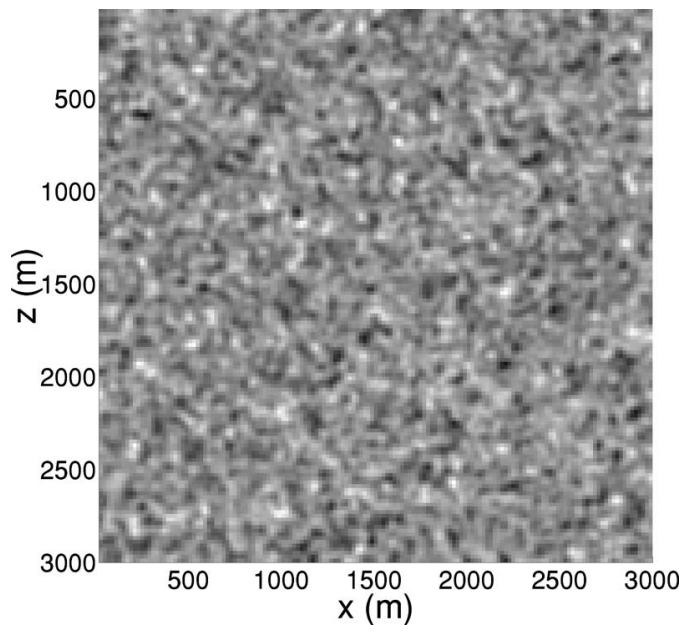

FIG. 8. 2D velocity model with random velocity fluctuations added to it. The correlation length of the velocity fluctuations is $40 \mathrm{~m}$.

distance $a$, with zero mean and standard deviation $\sigma$ (see Fig. 8 for a realization of the velocity model). The autocorrelation function of the velocity fluctuations $v_{r}$ has the form

$$
\left\langle v_{r}\left(\mathbf{r}^{\prime}\right) v_{r}\left(\mathbf{r}+\mathbf{r}^{\prime}\right)\right\rangle=\sigma^{2} \exp \left[\frac{-r^{2}}{a^{2}}\right] .
$$

The synthetic seismograms were created by transmitting a bandlimited pulse with a dominant wavelength of $240 \mathrm{~m}$. The grid size used was $20 \mathrm{~m}$ and the autocorrelation $a$ length was set to $40 \mathrm{~m}$, which is much smaller than the wavelength. The mean velocity $v_{0}$ is equal to $6000 \mathrm{~m} / \mathrm{s}$. To ensure strong scattering, we created a velocity field with a relative standard deviation of $25 \%$ about the mean velocity value. To test the validity of the diffusion approximation for our numerical model, we performed a numerical experiment where we placed a source in the middle of the model and recorded the seismograms on an array of receivers around the source. Figure 9 shows 100 synthetic seismograms computed at a distance of $250 \mathrm{~m}$ (left) and $3000 \mathrm{~m}$ (right) from the source. Note the strength of the multiple scattered arrivals after the highly attenuated ballistic arrival, especially for a distance of $3000 \mathrm{~m}$ from the source. The average intensity (which was

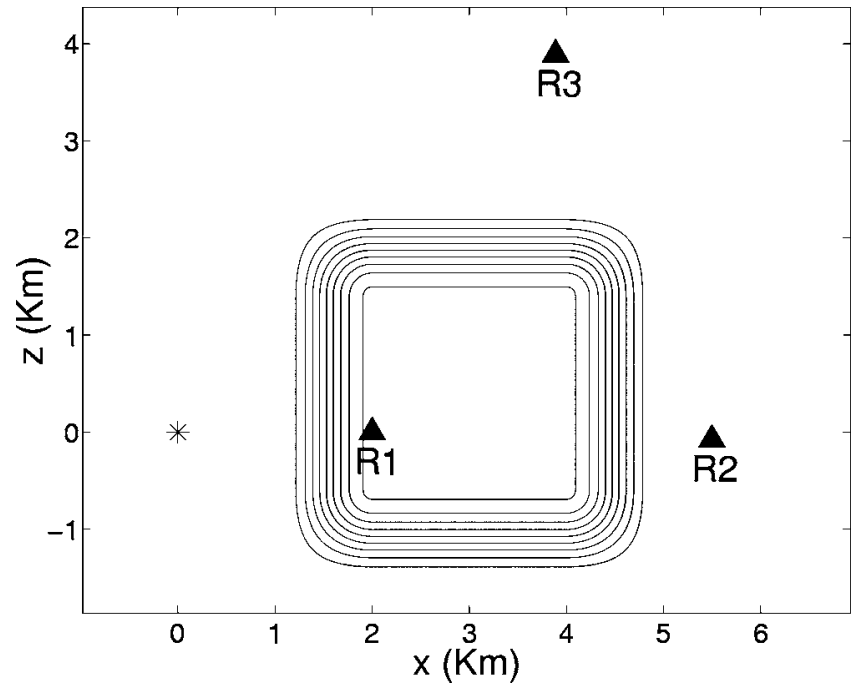

FIG. 10. Slowness perturbation added to the initial velocity model to create the perturbed velocity model. The side length of the square is $3000 \mathrm{~m}$ and the magnitude of the perturbation is $\delta s / s=0.005$. The source is shown as a star and the receivers as triangles.

shown in Fig. 1) was obtained by averaging the squared envelope of all calculated waveforms at a given distance to the source.

We test our theory with the finite-difference simulations of acoustic waves in the multiple scattering regime before and after a localized slowness perturbation has been introduced in the model. We perturb the random velocity model which represents the unperturbed medium by adding a localized slowness perturbation as shown in Fig. 10. The value of the relative slowness perturbation is $\delta s / s=0.005$.

We first analyze the unperturbed and perturbed synthetic seismograms for the receiver $\mathbf{R} 1$ located on the perturbed region of our model $2000 \mathrm{~m}$ away from the source. Figure 11 shows both the unperturbed and perturbed seismograms for receiver R1. The waveforms consist of diffuse or multiply scattered waves that have followed a multitude of paths from the source to the receiver. Notice the strength of the coda waves for late times. At first sight there seems to be no substantial difference between the wave field before and after the perturbation. However, zooming at around $2 \mathrm{~s}$ (see Fig.
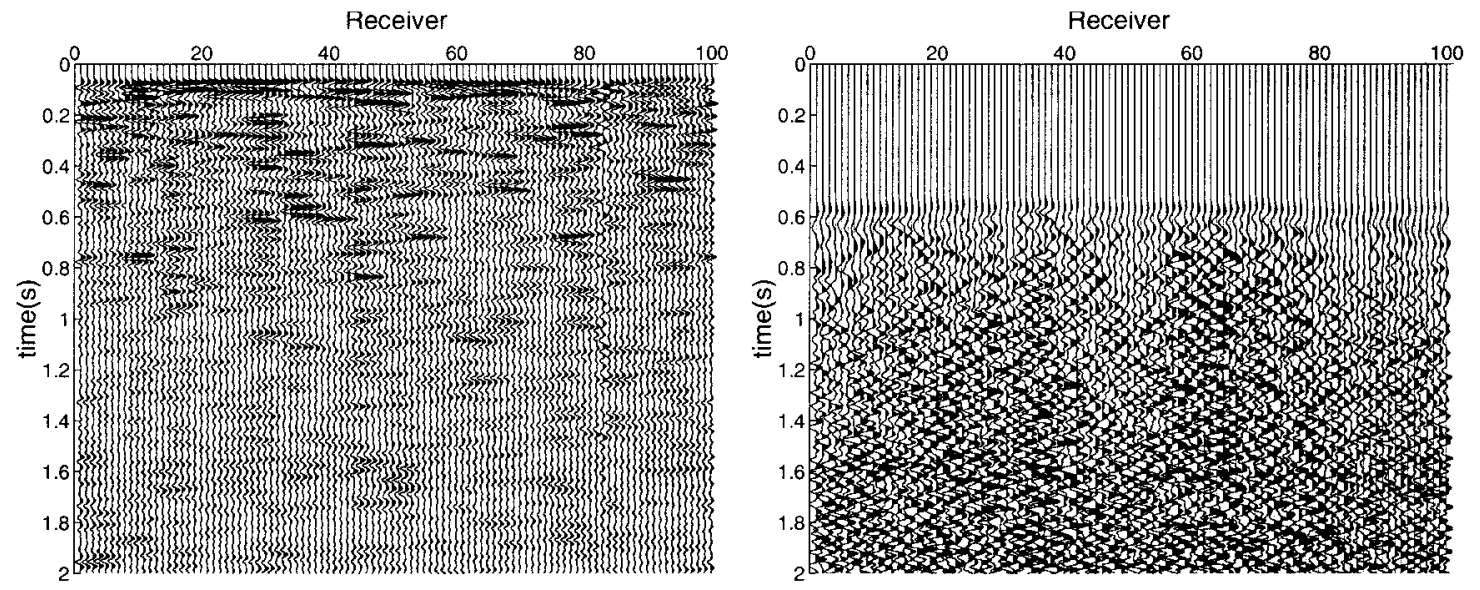

FIG. 9. Seismograms recorded at $250 \mathrm{~m}$ (left) and $3000 \mathrm{~m}$ (right) from the source. 


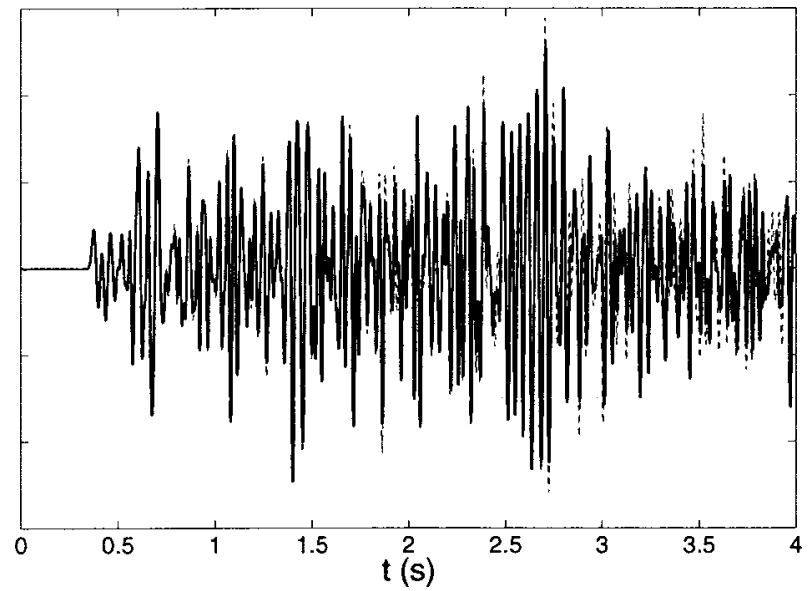

FIG. 11. Unperturbed (solid) and perturbed (dashed) synthetic seismograms recorded at the receiver $\mathbf{R} 1$ located $2000 \mathrm{~m}$ away from the source.

12) we see that the unperturbed seismogram lags in time with respect to the perturbed seismogram. More careful inspection indicates that the behavior of the time lags with travel time is systematic, i.e., the lag is increasing with travel time $t$.

We calculate the theoretical mean travel time change using Eq. (19) for receiver $\mathbf{R} 1$ at several propagation times $t$ and compare the result with the mean travel time change estimated from the synthetic seismograms using the timewindowed cross-correlation technique. Figure 13 shows good agreement between the theoretical and the measured mean travel time change $\langle\tau\rangle(t)$.

We also calculated the mean travel time changes for the receivers $\mathbf{R} 2$ and $\mathbf{R} 3$ located $5500 \mathrm{~m}$ away from the source. Even though the distance to the source is the same for these receivers, their locations with respect to the perturbation is different and therefore the mean travel time change is different for the two receivers. The theoretical travel time change for receiver $\mathbf{R} 2$ is almost twice the travel time change for receiver R3 (see Fig. 14). Also note that we have obtained a good agreement between the theoretical and estimated mean travel time change. This sensitivity of the mean travel time

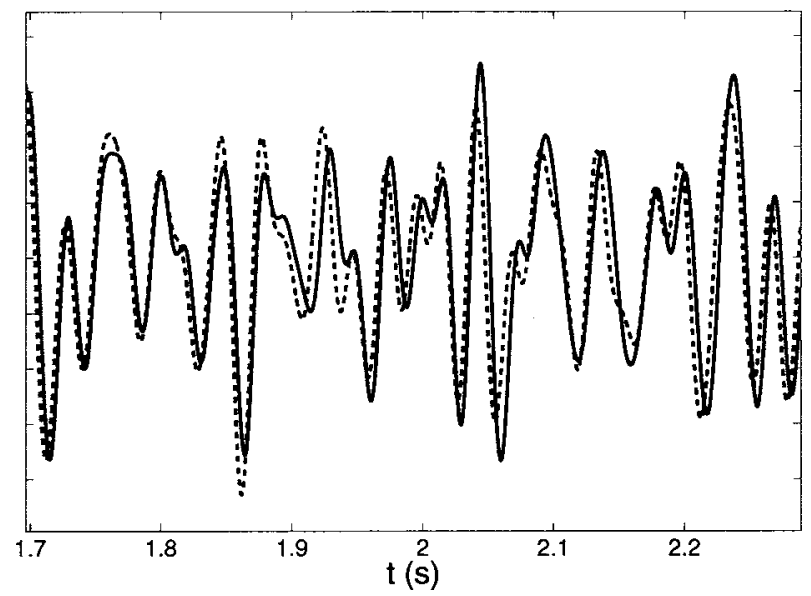

FIG. 12. Zoom of the unperturbed (solid curve) and perturbed (dashed curve) synthetic seismograms recorded at the receiver R1 located $2000 \mathrm{~m}$ away from the source. Note the time lags between the unperturbed and perturbed seismograms at different times.

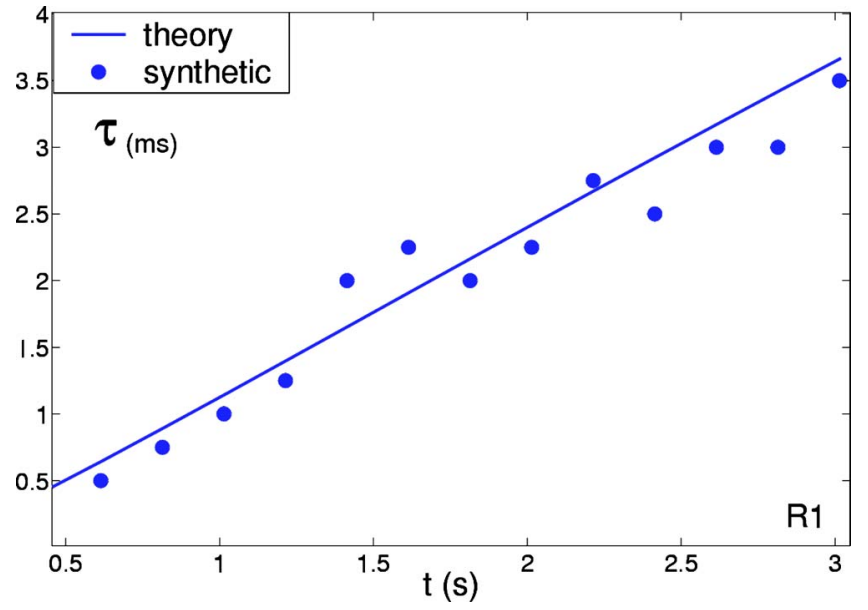

FIG. 13. (Color online) Theoretical versus measured mean travel time change for receiver R1 located $2000 \mathrm{~m}$ away from the source.

change to the relative source and receiver locations with respect to the perturbation can be exploited in an inversion scheme to estimate the propagation velocity change from measurements of the mean travel time change at different receiver locations. Notice also that for all receivers there are fluctuations of the measured travel time change about the theoretical value. We explore the origin of these fluctuations and how to minimize them in the following.

\section{FLUCTUATIONS OF THE MEAN TRAVEL TIME CHANGE}

We measured the mean travel time change from the synthetic seismograms using a time-windowed cross-correlation technique. The measured mean travel time change measured in this way is approximately an average of the time lags for the many scattering events on that time window. The fluctuations seen on the estimated mean travel time change are due to a number of factors, of which the most important is the size of the time window. We measure time lags of a continuous signal. Special care must be taken not to choose too small a time window to ensure stability of the estimation in the time-windowed cross correlation. The larger the time

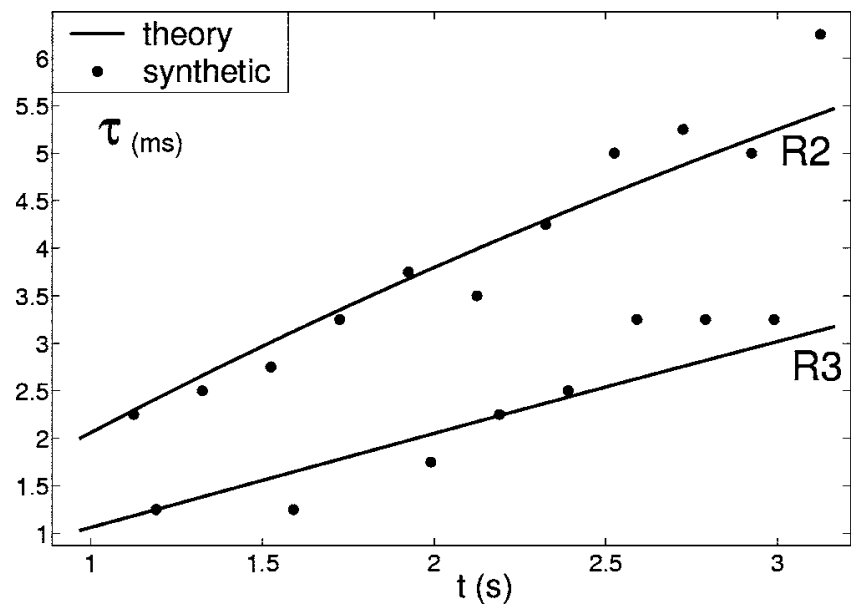

FIG. 14. Theoretical versus measured mean travel time change for receivers $\mathbf{R} 2$ and $\mathbf{R 3}$ located $5500 \mathrm{~m}$ away from the source. 

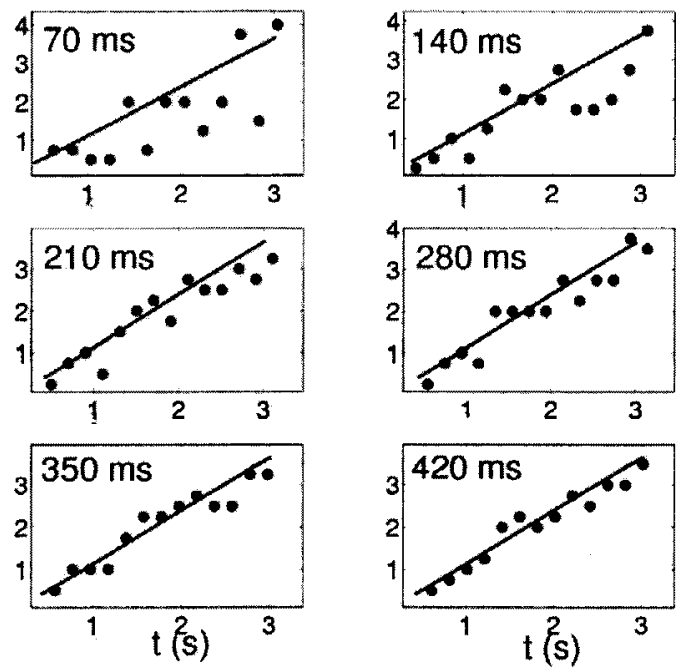

FIG. 15. Mean travel time change $\langle\tau\rangle(t)$ estimated from the synthetic seismograms for receiver $\mathbf{R} 1$ using different window lengths on the cross correlation. The mean travel time change is in milliseconds. Note the reduction in the fluctuations of the measured mean travel time change (circles) around the theoretical value (solid line) for larger window lengths. The dominant period is $40 \mathrm{~ms}$. The window length is shown in the upper-left corner of each plot.

window the more averaging of travel time perturbations occurs. Thus, choosing a larger window may help minimize the fluctuations of the observed mean travel time change.

We can reduce the fluctuations of the estimated mean travel time change $\langle\tau\rangle(t)$ by choosing a larger time window length $2 t_{w}$ on the time-windowed cross correlation. Reference 25 shows that increasing the time window length decreases the magnitude of the cross terms on the average intensity $\langle I\rangle$, and therefore the fluctuations of the time lags measured in the cross correlation. Larger contributions from the cross terms to the average intensity imply larger departures from the diffusive behavior.

The decrease of the fluctuations with the increase of the time window length $2 t_{w}$ can be appreciated in Fig. 15, where we plot the measured mean travel time change (circles) versus the theoretical mean travel time change (solid line) using different window lengths on the time-windowed cross correlation for the receiver $\mathbf{R} \mathbf{1}$ of example 1 of the previous section. We see that the fluctuations of the measured travel time change decrease with increasing window length $2 t_{w}$. For this synthetic example, the dominant period $T_{\text {dom }}$ is $40 \mathrm{~ms}$. The length of the time window in Fig. 15 varies from 70 to $420 \mathrm{~ms}$.

\section{CONCLUSIONS}

We have developed a technique that relates the mean travel time changes to the local changes in the propagation velocity of the medium. The theory is formulated by means of the diffusion approximation of the multiple scattering wave field. The sensitivity kernel $K(r, t)$ describes the dependence of the mean travel time change with the source and receiver location, the relative slowness perturbation, and the diffusion constant of the medium.

Equation (19) relates the mean travel time change $\langle\tau\rangle$ $\times(t)$ at time $t$ with a localized slowness perturbation $\delta s / s(\mathbf{r})$

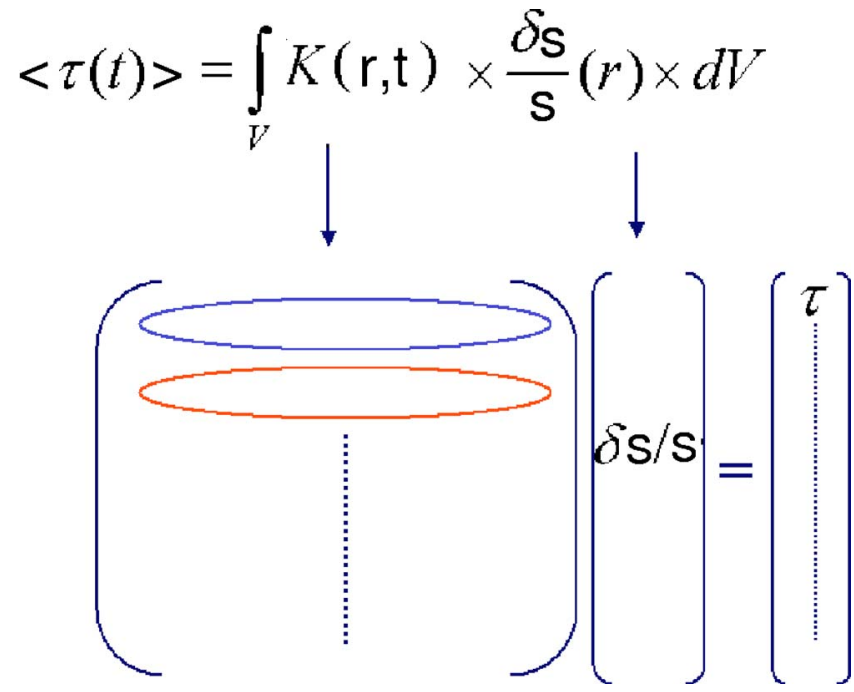

FIG. 16. (Color online) Schematic view of the tomographic problem in matrix form. The sensitivity kernel $K$ relates linearly the unknown parameters $\delta s / s$ with the measured traveltime change $\langle\tau\rangle(t)$. Each row in the matrix operator corresponds to a different receiver location.

Note that this equation can be used as the basis of a standard linear inverse problem, as there is a linearized relation between the data (mean travel time change for different times $t$, and different source/receiver pairs) and the unknown parameters of the medium (localized slowness perturbation) which we want to retrieve. In the previous section we showed that the mean travel time change is a function of time and of the source and receiver locations. This dependence of the mean travel time change on the relative source and receiver location with respect to the localized slowness perturbation can be exploited in the inversion scheme for the spatial location of the slowness change.

We have developed our technique for homogeneous media. In practice, detectors will most likely need to be placed on the surface of the medium, so that boundary conditions will have to be taken into account. Also, the medium may contain layers, so we will have to account for heterogeneity as well. The problem of analyzing the precise effect of a contrast of wave velocities and/or scattering strengths between them will arise. Under the assumption of an almost isotropic scattering field ${ }^{28}$ we determined the boundary conditions for the diffusion and radiative transfer equations for layered media. Undoubtedly all this will make our kernels more complicated.

Future work includes developing an inversion scheme to obtain the shape and magnitude of the slowness perturbation from the measured travel time changes at different receiver locations. This is similar to the transmission tomography problem, with the added complication that we are not only taking first or minimum time arrival but all multiple arrivals due to the interference of multiply scattered waves. The problem is simplified due to the fact that we are not using explicitly the paths as in transmission tomography but rather using the average wave field obtained with the diffusion approximation. Instead of calculating the travel time change with infinitely many line integrals, we calculate the mean travel time change using a much simpler volume integral that 
contains the contributions of all possible paths. For a given time $t$ and for a fixed source location we can set up the inverse problem in matrix form using different receivers as is shown in Fig. 16. The matrix operator (calculated from the time of flight distribution $K$ ) which multiplies the unknown parameter vector [slowness perturbation $\delta s / s(\mathbf{r})$ as a function of position] generates the data (mean travel time change for different receiver locations). Each row in the matrix corresponds to an observation of mean travel time change for a specific source-receiver pair at a discrete number of times $t$.

This technique can be used to detect temporal changes in highly heterogeneous material or reservoirs given that the diffusion approximation of the intensities is valid.

\section{ACKNOWLEDGMENTS}

This work was supported by the Consortium Project on Seismic Inverse Methods for Complex Structures at the Center for Wave Phenomena. We thank Ken Larner, Huub Douma, and Matt Haney for stimulating discussions.

\section{APPENDIX A: SENSITIVITY KERNEL $K$ FOR COINCIDENT SOURCE AND RECEIVER}

We start from the expression

$$
K\left(\mathbf{r}^{\prime}, \mathbf{s}, \mathbf{r}, t\right)=\int_{0}^{t} \frac{P\left(\mathbf{r}, \mathbf{r}^{\prime}, t-t^{\prime}\right) P\left(\mathbf{r}^{\prime}, s, t^{\prime}\right)}{P(\mathbf{r}, t)} d t^{\prime},
$$

where $P(\mathbf{r}, t)$ is the intensity at the receiver located at $\mathbf{r}$ due to a normalized impulse source at the origin at time $t=0$, and is given by Eq. (1). For homogeneous media it depends only on time and on the distance $r$ between source and receiver. In $2 \mathrm{D}$ it is equal to

$$
P(\mathbf{r}, t)=\frac{1}{4 \pi D t} \exp \left[\frac{-r^{2}}{4 D t}\right] .
$$

The time convolution is given by

$$
\begin{aligned}
P\left(\left|\mathbf{r}^{\prime}-\mathbf{s}\right|\right) * P\left(\left|\mathbf{r}-\mathbf{r}^{\prime}\right|\right)= & \int_{0}^{t} P\left(\left|\mathbf{r}^{\prime}-\mathbf{s}\right|, t^{\prime}\right) P\left(\left|\mathbf{r}-\mathbf{r}^{\prime}\right|, t\right. \\
& \left.-t^{\prime}\right) d t^{\prime}
\end{aligned}
$$

Substituting Eq. (A2) into Eq. (A3) gives for coincident source and receiver $(\mathbf{r}=\mathbf{s}=\mathbf{0})$

$$
P\left(\mathbf{r}^{\prime}, t\right)=\int_{0}^{t} \frac{\exp \left[\frac{-r^{\prime 2}}{4 D t^{\prime}}\right]}{4 \pi D t^{\prime}} \frac{\exp \left[\frac{-r^{\prime 2}}{4 D\left(t-t^{\prime}\right)}\right]}{4 \pi D\left(t-t^{\prime}\right)} d t^{\prime} .
$$

As there is symmetry around $t / 2$, we can write after renaming $\mathbf{r}^{\prime}$ as $\mathbf{r}$

$$
P(\mathbf{r}, t)=\frac{2}{(4 \pi D)^{2}} \int_{0}^{t / 2} \frac{\exp \left[\frac{-r^{2}}{4 D} \frac{t}{t^{\prime}\left(t-t^{\prime}\right)}\right] d t^{\prime}}{t^{\prime}\left(t-t^{\prime}\right)} .
$$

We apply the following changes of variables: $\epsilon$ $=1 /\left[t^{\prime}\left(t-t^{\prime}\right)\right]$ and then $\nu=r^{2} t\left[\epsilon-\left(4 / t^{2}\right)\right]$ to obtain

$$
P(\mathbf{r}, t)=\frac{2}{(4 \pi D)^{2}} e^{\left[-r^{2} / t\right]} \int_{0}^{\infty} \frac{e^{-\nu} d \nu}{\sqrt{\nu^{2}+\frac{\nu r^{2}}{D t}}} .
$$

This integral can be calculated identifying it as a integral of the type

$$
\int_{0}^{\infty} \frac{e^{-p x} d x}{\sqrt{x(x+a)}}=e^{a p / 2} K_{0}\left(\frac{a p}{2}\right),
$$

for $a>0$ and $p>0$. The solution to the integral in Eq. (A7) can be found in Ref. 29. Setting $p=1$ and $a=r^{2} / D t$, expression (A7) becomes

$$
(P * P)(\mathbf{r}, t)=\frac{2}{(4 \pi D)^{2} t} \exp \left[\frac{-r^{2}}{2 D t}\right] K_{0}\left[\frac{r^{2}}{2 D t}\right],
$$

where $K_{0}$ is the modified Bessel function of the second kind. Substituting Eqs. (A4) and (A2) into Eq. (A1), we arrive at the expression for the kernel $K(\mathbf{r}, t)$ in two dimensions for coincident source and receiver

$$
K_{2 D}(\mathbf{r}, t)=\frac{1}{2 \pi D} \exp \left[\frac{-r^{2}}{2 D t}\right] K_{0}\left[\frac{r^{2}}{2 D t}\right] .
$$

To obtain the sensitivity kernel $K$ in three-dimensional media, we substitute Eq. (A2) with the Green's function for the diffusion equation in $3 \mathrm{D}$

$$
P(\mathbf{r}, t)=\frac{1}{(4 \pi D t)^{3 / 2}} \exp \left[\frac{-r^{2}}{4 D t}\right] .
$$

Solving the integral defined in Eq. (A1) using the same changes of variables as in the 2D case, we obtain the expression for the kernel $K(\mathbf{r}, t)$ in three dimensions for coincident source and receiver

$$
K_{3 D}(\mathbf{r}, t)=\frac{1}{2 \pi D r} \exp \left[\frac{-r^{2}}{D t}\right] .
$$

${ }^{1} \mathrm{~J}$. Claerbout, Imaging the Earth's Interior (Blackwell, Cambridge, MA, 1985).

${ }^{2}$ J. P. Wesley, "Diffusion of seismic energy in the near range," J. Geophys. Res. 70, 5099-5106 (1965).

${ }^{3}$ Y. Kopnichev, "The role of multiple scattering in the formation of a seismogram's tail," Izv., Acad. Sci., USSR, Phys. Solid Earth 13, 394-398 (1977).

${ }^{4}$ S. A. Shapiro and G. Kneib, "Seismic attenuation by scattering: Theory and numerical results," Geophys. J. Int. 114, 373-391 (1993).

${ }^{5}$ J. Page, H. Schriemer, A. Bailey, and D. Weitz, "Experimental test of the diffusion approximation for multiply scattered waves," Phys. Rev. E 52(3), 3106-3114 (1995).

${ }^{6}$ H. Schriemer, M. Cowan, J. Page, Z. Liu, and D. Weitz, "Energy velocity of diffusing waves in strongly scattering media," Phys. Rev. Lett. 79(17), 3166-3191 (1997).

${ }^{7}$ A. Yodh and B. Chance, "Spectroscopy and imaging with diffusing light," Phys. Today 48, 34-40 (1995).

${ }^{8} \mathrm{G}$. $\mathrm{Li}$, "4D seismic monitoring of $\mathrm{CO} 2$ flood in a thin fractured carbonate reservoir," The Leading Edge 22(7), 690 (2003).

${ }^{9}$ T. Davis, M. Terrel, R. Cardona, R. Benson, R. Kendall, and R. Winarsky, "Multicomponent seismic characterization and monitoring of the $\mathrm{CO} 2$ flood at Weyburn Field, Saskatchewan," The Leading Edge 22(7), 696 (2003).

${ }^{10} \mathrm{~K}$. van Wijk, J. A. Scales, and J. Tromp, "Analysis of strong scattering at the microscale," J. Acoust. Soc. Am. 115(3), 1006-1011 (2004).

${ }^{11} \mathrm{~J}$. De Rosny and P. Roux, "Multiple scattering in a reflecting cavity: Application to fish counting in a tank," J. Acoust. Soc. Am. 109(6), 2587- 
2597 (2001).

${ }^{12}$ J. de Rosny, P. Roux, and M. Fink, "Field fluctuation spectroscopy in a reverberant cavity with moving scatterers," Phys. Rev. Lett. 90(9), 094302 (2003).

${ }^{13}$ P. Lemieux, M. Vera, and D. Durian, "Diffusing-light spectroscopies beyond the diffusion limit: The role of ballistic transport and anisotropic scattering," Phys. Rev. E 57(4), 4498-4515 (1998).

${ }^{14}$ D. Weitz and D. Pine, "Diffusing wave spectroscopy," in Dynamic Light Scattering, The Method and Some Applications, edited by W. Brown (Clarendon, Oxford, 1993), pp. 652-720.

${ }^{15}$ M. Cowan, I. Jones, J. Page, and D. Weitz, "Diffusing acoustic wave spectroscopy," Phys. Rev. E 65(066605), 1-11 (2002).

${ }^{16}$ R. Snieder, A. Grêt, H. Douma, and J. Scales, "Coda wave interferometry for estimating nonlinear behavior in seismic velocity," Science 295(22), 2253-2255 (2002).

${ }^{17} \mathrm{O}$. Lobkis and R. Weaver, "Coda-wave interferometry in finite solids: Recovery of P-toS conversion rates in an elastodynamic billiard," Phys. Rev. Lett. 90(25), 254302 (2003).

${ }^{18} \mathrm{M}$. Snieder and M. Vrijlandt, "Constraining relative source locations with coda wave interferometry: Theory and application to earthquake doublets in the Hayward Fault, California," J. Geophys. Res. (submitted).

${ }^{19}$ A. Tourin, A. Derode, and M. Fink, "Sensitivity to perturbations of a time-reversed acoustic wave in a multiple scattering medium," Phys. Rev. Lett. 87(27), 274301 (2001).

${ }^{20}$ A. Tourin, M. Fink, and A. Derode, "Multiple scattering of sound," Waves Random Media 10, R31-R60 (2000).
${ }^{21}$ J. Paasschens, "Solution of the time-dependent Boltzmann equation," Phys. Rev. E 53, 1135-1141 (1997).

${ }^{22}$ S. Skipetrov and R. Maynard, "Diffuse waves in nonlinear disordered media," in Wave Scattering in Complex Media: From Theory to Applications, Vol. 107 of Nato Science Series, edited by B. van Tiggelen and S. Skipetrov (Kluwer Academic, Dordrecht, 2003), pp. 75-97.

${ }^{23} \mathrm{R}$. Snieder, "Coda wave interferometry and the equilibration of energy in elastic media," Phys. Rev. E 66, 046615-046618 (2002).

${ }^{24} \mathrm{R}$. Snieder, "Imaging and averaging in complex media," in Diffuse Waves in Complex Media, edited by J. Fouque (Kluwer Academic, Raleigh, NC, 1999), pp. 405-454.

${ }^{25}$ R. Snieder, "Extracting the Green's function from the correlation of coda waves: A derivation based on stationary phase," Phys. Rev. E 69, 046610 (2004).

${ }^{26}$ G. Roepstoff, Path Integral Approach to Quantum Physics: An Introduction (Springer, New York, 1994).

${ }^{27}$ A. Frankel and R. Clayton, "A finite-difference simulation of wave propagation in two dimensional random media," Bull. Seismol. Soc. Am. 74(6), 2167-2186 (1984)

${ }^{28}$ L. Margerin, M. Campillo, and B. van Tiggelen, "Radiative transfer and diffusion of waves in a layered medium: New insight into coda q," Geophys. J. Int. 134, 596-612 (1998).

${ }^{29} \mathrm{~J}$. Gradshteyn and I. Ryzhik, in Table of Integrals, Series, and Products, 7th ed., edited by Y. V. Geronimus and M. Tseytlin (Academic, New York, 1973). 